\title{
Neutrino physics with accelerator driven subcritical reactors
}

\section{Emilio Ciuffoli, Jarah Evslin and Fengyi Zhao}

Institute of Modern Physics, CAS, NanChangLu 509, Lanzhou 730000, China

E-mail: emilio@impcas.ac.cn, jarah@impcas.ac.cn, fengchu@impcas.ac.cn

ABSTRACT: Accelerator driven system (ADS) subcritical nuclear reactors are under development around the world. They will be intense sources of free, $30-55 \mathrm{MeV} \mu^{+}$decay at rest $\bar{\nu}_{\mu}$. These ADS reactor neutrinos can provide a robust test of the LSND anomaly and a precise measurement of the leptonic CP-violating phase $\delta$, including $\operatorname{sign}(\cos (\delta))$. The first phase of many ADS programs includes the construction of a low energy, high intensity proton or deuteron accelerator, which can yield competitive bounds on sterile neutrinos.

Keywords: Oscillation, Neutrino Detectors and Telescopes, CP violation

ARXIV EPRINT: 1509.03494 


\section{Contents}

1 Introduction 1

2 Isotope decay at rest experiment $\quad 3$

3 Muon decay at rest experiment $\quad 6$

$\begin{array}{lll}3.1 & \text { Sterile neutrino appearance experiment } & 7\end{array}$

3.2 CP violation 8

4 Conclusions 10

\section{Introduction}

With their ability to enrich spent fuel and their inability to melt down, accelerator driven system (ADS) subcritical reactors are being designed and prototypes are being built around the world. ADS machines in general will be powered by proton beams, of energies of 1$2 \mathrm{GeV}$ with currents of 10-20 mA, which strike high $Z$ targets. Beam energies of $400 \mathrm{MeV}$ or more lead to the production of pions. The $\pi^{-}$are generally absorbed in the target, while $\pi^{+}$stop and decay at rest (DAR), producing $\mu^{+}$which also stop and decay at rest, producing $\bar{\nu}_{\mu}$ with a known energy spectrum peaked between 30 and $55 \mathrm{MeV}$. Like the $\bar{\nu}_{e}$ from ordinary critical reactors, these $\bar{\nu}_{\mu}$ are free byproducts of the reaction, requiring no modification to the reactor design and so are at no cost to the neutrino physicist. Early phases of ADS use less energetic beams which can produce $\bar{\nu}_{e}$ via the decay at rest of spallation isotopes (IsoDAR). In this note we describe some of the neutrino physics which can be done with these ADS reactor neutrinos.

For example, in Europe the MYRRHA ADS research reactor has been approved. Its proton accelerator is designed to operate at $600 \mathrm{MeV}$ with a current of $4 \mathrm{~mA}$, and so it will already be a powerful source of $\mu \mathrm{DAR} \bar{\nu}_{\mu}$.

In this paper we will consider two configurations which are currently planned by the Chinese ADS (C-ADS) collaboration [1]. The first is a prototype called CI-ADS which consists of a deuterium beam with $100 \mathrm{MeV} /$ nucleon and a current of $15 \mathrm{~mA}$. The second, corresponding to C-ADS phase II, is an $800 \mathrm{MeV}$ proton beam with a current of $10 \mathrm{~mA}$. As the beam energy in the first setup is below $400 \mathrm{MeV}$, it cannot provide $\mu \mathrm{DAR} \bar{\nu}_{\mu}$ and so will be used for a $\bar{\nu}_{e}$ program which will constrain the sterile neutrino mixing angle $\theta_{e e}$. The second will use $\bar{\nu}_{\mu}$ to constrain the sterile neutrino mixing angle $\theta_{\mu e}$, providing a robust test of the LSND anomaly, and to measure the leptonic CP-violating phase $\delta$.

These experimental goals and their implementations are inspired by the DAE $\delta$ ALUS project [2]. However there are several key differences. DAE $\delta$ ALUS uses multiple accelerators with various baselines to the detector. To determine which event came from which 
source, it requires that only one source runs at a time. Therefore the maximum instantaneous current at each DAE $\delta$ ALUS source needs to be quite high: for the far source they envisage 30-50 mA. On the other hand, ADS projects consist of a single source and so the beam can be truly continuous and the same number of events/baseline can be achieved with a much lower maximum instantaneous current. Second, DAE $\delta$ ALUS proposes the construction of its own cyclotrons; this is necessary since no country's ADS program envisages the construction of multiple machines with the $10-20 \mathrm{~km}$ separation required by their proposal. On the other hand, the C-ADS proposal already envisages the construction of the accelerators and, for the $\mu \mathrm{DAR}$ experiment, also the target station required by our proposal. Third, as a commercial reactor ADS has a highly redundant design. Even the C-ADS injecting accelerators are redundant. This redundancy will allow ADS to have very little downtime. Finally, in the case of C-ADS, the location in southern China has the advantage that the horizontal component of the Earth's magnetic field is $0.38 \mathrm{G}$, as compared with $0.17 \mathrm{G}$ at the original DAE $\delta$ ALUS location in South Dakota and $0.13 \mathrm{G}$ at the second DAE $\delta$ ALUS location in the Pyhäsalmi mine. As a result the main backgrounds, which arise from low energy atmospheric neutrinos, are reduced by about a factor of two in our setup.

The MOMENT experiment [3] also intends to use ADS technology to measure the CPviolating phase $\delta$. While the high intensity beam is similar, there are several key differences between MOMENT and the current proposal arising from the fact that MOMENT uses decay in flight $\mu^{+}$to generate its neutrinos whereas our proposal uses decay at rest. One consequence is that our neutrino energies are much lower. This implies that we detect our signal using inverse $\beta$ decay (IBD), which has a very precisely known cross-section, whereas MOMENT will use quasielastic charged current interactions, whose cross-section is larger but is known much less precisely in the relevant energy range. A second consequence is that at our neutrino energies, $\nu_{\mu}$ have no charged current interactions and thus do not contribute to the background in our experiment. Also the $\mu \mathrm{DAR} \nu$ energy spectra are known precisely, whereas MOMENT's decay in flight spectrum is estimated by measuring the properties of the beam. Finally, MOMENT requires a beam energy well in excess of $1 \mathrm{GeV}$, and so must await the completion of the full ADS project.

Perhaps the most important difference between the $\mu$ DAR proposal and MOMENT is that $\mu \mathrm{DAR}$ occurs automatically in ADS target stations. Therefore a $\mu \mathrm{DAR}$ experiment in no way interferes with the operation of an ADS reactor. On the other hand, MOMENT requires a beam of $\pi$ to be extracted from the target station. It is unlikely that this can be done in a working ADS reactor, and so MOMENT suggests the construction of a new, dedicated, ADS-like accelerator for its experiment.

On the other hand, the first phase of our proposal, IsoDAR, does require a custom made target station on the CI-ADS accelerator. The ADS research program requires that the accelerator is frequently tuned and modified with the goal of enhancing the beam stability, which will considerably reduce the live time but we believe that we can more than compensate for this loss via optimizations in our target design which will be explained in a followup paper. The target station is planned 50 to 100 meters away from the accelerator itself, with the detectors on the side and slightly upstream, and so no interference with the accelerator operations is foreseen. 
We note that there is already an extensive literature on the use muon neutrinos from spallation sources for sterile neutrino searches [4] and to measure the CP-violating phase $\delta[5]$. ADS envisages beams which are an order of magnitude more powerful than spallation sources $^{1}$ and yet applications of neutrinos from ADS have so far received little attention. The current paper aims to begin filling this gap.

\section{Isotope decay at rest experiment}

In the first experimental setup, which is similar to that proposed in refs. [7, 8], we use the $100 \mathrm{MeV} /$ nucleon deuterium beam to knock neutrons out of ${ }^{9} \mathrm{Be}$. These neutrons are moderated and multiplied by heavy water moderator and then absorbed by $99.99 \%$ isotopically pure ${ }^{7} \mathrm{Li}$, producing ${ }^{8} \mathrm{Li}$. The eventual $\beta$ decay of the ${ }^{8} \mathrm{Li}$ produces our $\bar{\nu}_{e}$ with an energy spectrum peaked at $6 \mathrm{MeV}$ but extending up to $13 \mathrm{MeV}$. These $\bar{\nu}_{e}$ travel isotropically and are eventually detected via the inverse beta decay reaction

$$
\bar{\nu}_{e}+p \rightarrow n+e^{+}
$$

on hydrogen in liquid scintillator detectors placed at distances between 5 and 30 meters. Neutrino oscillations between the three known flavors are inappreciable at distances below 100 meters, thus a deficit of detected $\bar{\nu}_{e}$ suggests oscillations to sterile neutrinos.

Backgrounds are notoriously problematic in such experiments, and so it is essential to place multiple detectors at multiple baselines. The baseline-dependence of the neutrino disappearance is well-known for a single flavor of sterile neutrino and so can be used both to confirm that the anomaly is truly caused by sterile neutrinos and also to break the degeneracy between the sterile neutrino mixing angle and the sterile neutrino mass splitting. In the case of C-ADS, obtaining multiple detectors is in principle quite simple. C-ADS will be located in China's Guangdong province. In the same province, Daya Bay's eight 20 ton liquid scintillator detectors will no longer be needed. We therefore suggest using as many of these eight Daya Bay detectors as possible, although in our simulations we have conservatively estimated that only two will be available.

The near site neutrino detectors also provide useful information for the ADS team as a real-time target monitoring system.

The beam-off backgrounds in this experiment are quite well understood, as they are identical to those in current reactor experiments. They are essentially all in some way created by cosmogenic muons and so can be considerably reduced with vetoes near these muon events and a low energy veto. We veto all events with reconstructed neutrino energies beneath $6 \mathrm{MeV}$. We include the background from decays of spallation ${ }^{9} \mathrm{Li}$, which is so long lived that it cannot be effectively removed by muon vetoes at our depth. We have assumed a depth of 100 meters, which has been considered for the target station. For a 20 ton LABbased scintillator detector, this gives us 3 background events/detector/day, consistent with that observed by the Daya Bay experiment with a similar overburden.

\footnotetext{
${ }^{1}$ Potentially more powerful pulsed beams accelerated by lasers may be used for both kinds of neutrino experiment $[6]$.
} 
CI-ADS will be built in the Huizhou county of Guangdong province. In Guangdong, 14 nuclear reactors are either operational or under construction. The nearest, at Daya Bay and Ling Ao, are about $48 \mathrm{~km}$ from the leading candidate site. As a result, the reactor neutrino background will be about 0.1 events/day. Another reactor complex has been proposed in Lufeng, but it is about the same distance and power as the Daya Bay and Ling Ao complex and so would only contribute another 0.1 event/day. There have however been proposals to build a reactor complex in Huizhou, $2 \mathrm{~km}$ southwest of the favored CI-ADS site. As it requires about five years to build a reactor in China and then usually one reactor is completed per year, it is unlikely that any reactors will be built before CI-ADS.

However it remains possible that the first reactors will come on line while the IsoDAR experiment is taking data. In this case the reactor background will certainly exceed the spallation background. If 6 reactors are eventually built at this site, then reactor neutrinos would contribute roughly 70 background events per day per detector, of which 10 would satisfy our $6 \mathrm{MeV}$ veto. This background can be measured while our beam is off and calibrated to the status of the reactor complex, using weekly updates supplied by the power company. About half of this background can be eliminated by increasing the low energy veto to $7 \mathrm{MeV}$.

Although we have run a series of GEANT 4 simulations of the target, described in ref. [9], we have not considered beam-on backgrounds in this analysis. Preliminary results indicate however that the main beam-on backgrounds will arise from neutrons that escape the target station and neutrinos from other decays. The first is greatly reduced by using shielding and by placing the detectors far from the beam line, and so we intend to place them somewhat upstream from the target station. The latter will be clustered at low energies and will likely be largely removed by the energy cut.

The disappearance signal resulting from a single family of sterile neutrino, as a function of baseline, is well-known once one fixes the mixing angle and mass splitting. Backgrounds from neutrons fall much faster with distance. Therefore, by including more detectors one measures the disappearance at more baselines and so can fix the mixing angle and mass splitting parameters and simultaneously distinguish the signal from the neutron background. On the other hand, with only two detectors, a background which leads to a higher ratio of near to far detector events could be difficult to distinguish from a sterile neutrino induced disappearance. In all, the Daya Bay experiment has eight detectors which may be available for our use. The use of more than two detectors will strengthen the estimation of the background.

We consider a run with $5 \times 10^{23}{ }^{8} \mathrm{Li}$ decays, each yielding an $\bar{\nu}_{e}$. In the absence of sterile neutrinos this yields $2 \times 10^{4}$ IBD events in a 20 ton target volume with $12 \%$ free protons at a distance of 30 meters. We assume a $5 \%$ uncertainty on this event rate and a $1 \%$ error per $50 \mathrm{keV}$ bin on the background rate. As we have multiple detectors, the final precision depends only weakly upon this uncertainty unless $\Delta M^{2}<0.3 \mathrm{eV}^{2}$, which is well outside the region preferred by global fits of sterile neutrino data.

Note that, since the ${ }^{8} \mathrm{Li}$ spectrum is independent of the beam and target station parameters, for any deuterium beam energy and current and any target configuration, one may obtain $5 \times 10^{23}$ such decays for an experiment of some duration. Therefore, given any 
beam and target parameters, one need only estimate this run time. Neutron production rates from $62 \mathrm{MeV}$ and $200 \mathrm{MeV}$ deuteron beams on ${ }^{9}$ Be have been measured in refs. $[10,11]$ where they were found to yield roughly 0.12 and 0.6 neutrons/deuteron respectively. As has been stressed in ref. [8], a deuterium beam is advantageous in that it directly brings neutrons to the target, which are liberated via proton stripping and deuterium breakup. The deuterium breakup fast neutrons have energies peaked around half of the deuterium beam energy and so they must travel a long distance before slowing enough to have a high interaction cross section with ${ }^{7} \mathrm{Li}$. This places strong constraints on the target geometry. For this purpose we have considered a target with multiple ${ }^{7} \mathrm{Li}$ sleeves surrounded by a graphite reflector. Our GEANT 4 simulations indicate that nonetheless only about $6 \%$ of the neutrons are captured by ${ }^{7} \mathrm{Li}$ in our particular target geometry. This means that we require $8 \times 10^{24}$ neutrons and so $1.4 \times 10^{25}$ deuterons on target. With a $15 \mathrm{~mA}$ beam this corresponds to 5 years of running. Preliminary simulations show that an optimization in the target geometry as in ref. [12], so as to increase the neutron capture rate, considerably reduces this run time.

We approximate the fractional energy resolution of the Daya Bay detectors, 5 years from now, to be

$$
\sigma=\sqrt{\left(\frac{7 \%}{\sqrt{E}}\right)^{2}+(1 \%)^{2}} .
$$

We assume that all of the neutrinos arrive from a single point in the center of the target. In practice they will be smeared with a characteristic size of order one meter which will appreciably reduce our sensitivity to sterile neutrinos with $\Delta M^{2}$ of order $10 \mathrm{eV}^{2}$ or more. However this smearing depends strongly on the design of our target station which is not yet fixed. As most neutrons exit the target in a fairly narrow cone, a detector location perpendicular to the beamline minimizes the scatter in the neutrino baselines.

We have parametrized the $\bar{\nu}_{e}$ disappearance using the parameters $\theta_{e e}$ and $\Delta M^{2}$ defined by

$$
P_{e e}=1-\sin ^{2}\left(2 \theta_{e e}\right) \sin ^{2}\left(1.27 \frac{\Delta M^{2} L}{E}\right)
$$

where $L$ is the baseline in meters and $E$ is the neutrino energy in $\mathrm{MeV}$. We have determined the parameter values which may be excluded at $2 \sigma, 3 \sigma$ and $5 \sigma$ for all values of pairs of baselines between 5 and 30 meters in a 5 year run, using the crude approximation that the sources and detectors are point-like. As the detectors can reconstruct the positions of events with a precision of about $10 \mathrm{~cm}$, the point-like detector approximation has a much smaller effect on the sensitivity than the point-like source approximation discussed above.

Fixing the near and far detector baselines to be $5 \mathrm{~m}$ and $15 \mathrm{~m}$, our exclusions region is shown in figure 1. Already in this 2-detector setup, in the $0.5 \mathrm{eV}^{2}-10 \mathrm{eV}^{2}$ range this exclusion regime is competitive with the most sensitive proposed sterile neutrino experiments. This proposal is complimentary to reactor-based experiments (reviewed in ref. [14]) that will measure $\theta_{e e}$ because the ${ }^{8} \mathrm{Li}$ decay spectrum extends about $50 \%$ higher than the relevant part of the reactor spectrum, and so one has access to the same values of $L / E$ 


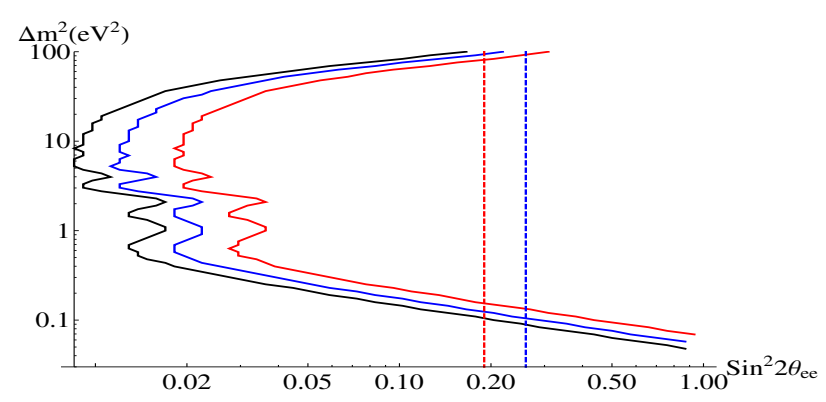

Figure 1. The region of $\theta_{e e}-\Delta M^{2}$ parameter space which may be excluded with $2 \sigma$ (black curve), $3 \sigma$ (blue curve) and $5 \sigma$ (red curve) of confidence by an IsoDAR run with a near detector at $5 \mathrm{~m}$ and a far detector at $15 \mathrm{~m}$. The red and blue vertical lines are the 2 degree of freedom $95 \%$ exclusion limits from long baseline experiments and from solar neutrinos plus KamLAND respectively, as summarized in ref. [13].

with values of $L$ and $E$ which are nonetheless distinct from those so far probed, providing an additional degree of robustness.

\section{Muon decay at rest experiment}

In the rest of this paper we will consider C-ADS Phase II with a $10 \mathrm{~mA}$ beam of $800 \mathrm{MeV}$ protons. This accelerator creates neutrinos via $\pi^{+}$and $\mu^{+}$DAR

$$
\pi^{+} \rightarrow \mu^{+}+\nu_{\mu}, \mu^{+} \rightarrow e^{+}+\nu_{e}+\bar{\nu}_{\mu}
$$

The $\bar{\nu}_{\mu}$ spectrum of $\mu \mathrm{DAR}$ neutrinos is well known. To determine the normalization of the neutrino signal we again suggest using several near detectors, such as Daya Bay detectors, now at baselines ranging from $30 \mathrm{~m}$ to $100 \mathrm{~m}$ from the target. The results that we report below use two Daya Bay detectors, one at $50 \mathrm{~m}$ and one at $100 \mathrm{~m}$.

The near detectors can determine the normalization using neutrino-electron elastic scattering. To determine the expected elastic scattering event rate, we simply scale the measurements of LSND, as the neutrino spectra have the same shape. With $1.8 \times 10^{23}$ protons on target (POT), LSND estimates that it observed about 250 elastic scattering events with a 167 ton detector at a baseline of 30 meters. The target volume is $97 \% \mathrm{CH}_{2}$ and so $Z / A=0.57$. In 5 years of live time with a $10 \mathrm{~mA}$ beam we will have $10^{25}$ protons on target. Multiplying by the ratio of the protons on target and detector volumes and dividing by the square of the ratio of baselines, a 20 ton $(Z / A=0.56)$ detector at $50(100)$ meters will therefore observe 530 (133) elastic scattering events.

In addition to elastic scattering events, there are also quasielastic (CCQE) ${ }^{12} C\left(\nu_{e}, e^{-}\right) N_{\text {g.s. }}$ events, which are about three times as frequent, have a similar spectrum and also result in the production of an electron. These can also be used to fix the $\mu \mathrm{DAR}$ neutrino flux normalization although their spectrum is more uncertain. For a more precise measurement, one would like to distinguish the signals from these two processes. LSND was able to cleanly distinguish these because the CCQE events produce isotropically distributed electrons whereas essentially all of the elastic scattering electrons move within a 


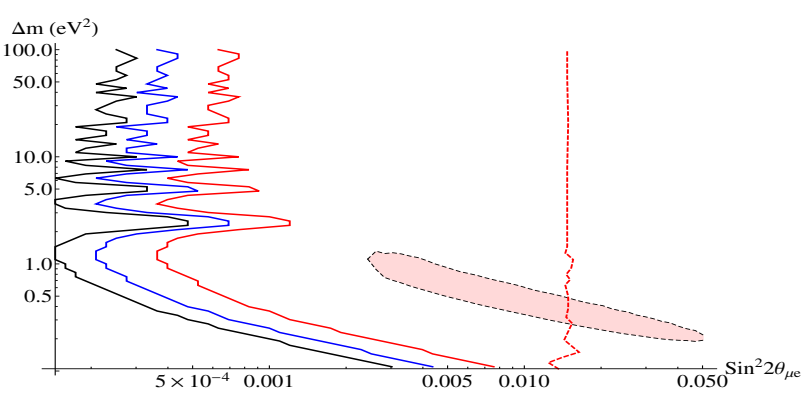

Figure 2. The region of $\theta_{\mu e}-\Delta M^{2}$ parameter space which may be excluded with $2 \sigma$ (black), $3 \sigma$ (blue) and $5 \sigma$ (red) of confidence by a 5 year $\mu$ DAR run with a near detector at $50 \mathrm{~m}$ and a far detector at $100 \mathrm{~m}$. The red dashed line is the $99 \%$ exclusion limit from the ICARUS experiment as reported in ref. [16] while the pink region is the preferred sterile neutrino region from a combination of LSND and MiniBooNE [13].

20 degree cone pointed away from the neutrino source. The electron direction was easily determined at LSND as the detector was $97 \%$ mineral oil, which led to a clear Cherenkov cone. The separation capability that can be obtained employing the Daya Bay detectors is under evaluation.

\subsection{Sterile neutrino appearance experiment}

In this experiment, the near detectors will again play the dual role of a sterile neutrino search and a real time monitor of the target. However, the sterile neutrino search will now be based on the $\bar{\nu}_{\mu} \rightarrow \bar{\nu}_{e}$ appearance channel. Thus it will not be sensitive to $\theta_{e e}$ but rather to $\theta_{\mu e}$, defined by

$$
P_{\mu e}=\sin ^{2}\left(2 \theta_{\mu e}\right) \sin ^{2}\left(1.27 \frac{\Delta M^{2} L}{E}\right)
$$

and so it will test of the LSND anomaly [15]. Like LSND, as an appearance experiment its reach will extend to much lower values of the relevant mixing angle than a disappearance experiment. In fact, as the proton current is an order of magnitude greater than LSND and as there are multiple detectors, it will provide a much more robust test of this anomaly than LSND itself, ranging down to lower mass splittings and smaller mixing angles.

The $2 \sigma, 3 \sigma$ and $5 \sigma$ bounds which may be achieved on $\theta_{\mu e}$ and $\Delta M^{2}$ in a 5 year run are presented in figure 2. Note that the best fit LSND anomaly region is well covered. Elastic $e-\nu$ scattering in the near detectors can be used to determine the $\bar{\nu}_{\mu}$ flux with an uncertainty of about $5 \%$. However the impact of this uncertainty on the exclusion limits is negligible. The beam-off backgrounds are dominated by atmospheric neutrinos which will be relevant at the far detectors but at the near detectors yield backgrounds which are again negligible. The beam-on backgrounds are more difficult to anticipate, but we expect a minor contribution in this energy range. Following the estimates given by LSND [15] we fix the beam-on background by asserting that for each $\mu^{+}$DAR there are $5 \times 10^{-4} \mu^{-}$ DARs and that the normalization of this background is known with a precision of $25 \%$. We find that this beam-on background has little effect on our results. The $\mu^{-}$are created 
by $\pi^{-}$which decay in flight and, as we will explain below, we expect much less $\pi^{-}$to decay in flight than at LSND and so this background estimate is conservative.

At the LSND experiment, there was also a significant contribution to the $\bar{\nu}_{\mu}$ from the decay in flight (DIF) of $\pi^{-}$. These have typical energies of about $50 \mathrm{MeV}$, but extend in energy up to about $200 \mathrm{MeV}$. Therefore some of these yield a false signal via the charged current interaction

$$
\bar{\nu}_{\mu}+p \longrightarrow \mu^{+}+n \text {. }
$$

ref. [15] estimated the ratio of these events to the $\mu^{+}$DAR signal, assuming $100 \% \bar{\nu}_{\mu} \rightarrow \bar{\nu}_{e}$ oscillation, to be $3 \times 10^{-4}$, corresponding to about $12 \%$ of the observed excess. Therefore this background was about half of the $\mu^{-}$DAR background, although with a poorly known energy spectrum.

Note that the $\pi^{+}$DIF background is at least five times larger as the $\pi^{+} / \pi^{-}$ratio at LSND is about 8 and $3.4 \%(5 \%)$ of $\pi^{+}\left(\pi^{-}\right)$decay in flight [17], but the resulting $\nu_{\mu}$ only produce neutrons in those interactions with ${ }^{12} \mathrm{C}$ that lead to particular final state interactions. In ref. [18] it was estimated that the $\nu_{\mu}$ flux above the muon production threshold on carbon exceeds that of $\bar{\nu}_{\mu}$ by more than a factor of eight. However at LSND it is possible to identify the final state muon from its eventual decay so long as it has sufficient energy and decays within $12 \mu \mathrm{sec}$. Furthermore, these interactions have a higher threshold than (3.3) because of the nuclear binding energy of the ejected nucleon, an effect which is compounded by the low energy veto at LSND. Vetoing events with identified muons and requiring at least $20 \mathrm{MeV}$ of observed energy, LSND finds [15] that this background is a factor of six smaller than that of (3.3). Daya Bay detectors are sensitive to even lower energies and so this rejection efficiency can only improve. In fact, we expect the $\pi$ DIF rate to be significantly smaller in the experiment under consideration.

At LSND, the $\pi^{-}$DIF background largely came from $\pi^{-}$which decay in $50 \mathrm{~cm}$ of airfilled decay region located between the target station and the isotope production targets. About $6 \%$ came from other experiments at the two target stations located about $100 \mathrm{~m}$ upstream from the target used by LSND [18]. In the case of ADS, the design currently includes neither a decay region nor other upstream targets. The $\pi$ will enter directly into a moderator which will slow and capture nearly all of the $\pi^{-}$before they can decay. Although the composition of the moderator has not been fixed, it will not be a dilute gas. Therefore, as in the case of the LSND isotope production target and beam dump, the rate of $\pi^{-}$decay in flight is expected to be negligible.

\subsection{CP violation}

The physics program described above can be performed at very moderate cost, as the $\bar{\nu}$ have been supplied for free by ADS, albeit with a modified target station in the first setup, and the detectors have been recycled from Daya Bay. However perhaps the most exciting potential application of ADS reactor neutrinos is the measurement of the leptonic CP-violating phase $\delta$. For this purpose, we will consider two 20 kton liquid scintillator detectors at baselines of $2.5-30 \mathrm{~km}$ to detect $\bar{\nu}_{e}$ created by $\bar{\nu}_{\mu}$ oscillations. Combining this appearance result with $\nu_{\mu} \rightarrow \nu_{e}$ from $\mathrm{NO} \nu \mathrm{A}$ and $\mathrm{T} 2 \mathrm{~K}$ one obtains the leptonic CPviolating phase $\delta$. Unlike the 20 kton detectors needed for mass hierarchy experiments, 
the measurement of $\delta$ does not impose stringent restrictions upon the detector's energy resolution or the knowledge of its nonlinear energy response. Thus, unlike JUNO [19], the detector can be cylindrical, the PMT coverage can be less than $50 \%$ and the scintillator purity does not need to exceed that in present day experiments. Also, as $80 \%$ of the events are at energies above $30 \mathrm{MeV}$ and so well above the spallation isotope backgrounds, the detectors do not need to be as deep as JUNO.

Such a setup has several advantages for measuring $\delta[20,21]$. Not only is the $\bar{\nu}_{e}$ spectrum well known, but since they are detected via IBD the cross section is also well known and the capture of the resulting neutron provides a double coincidence which can be used for background rejection. As a $\bar{\nu}$ oscillation experiment it maximizes synergy with beam experiments such as $\mathrm{T} 2 \mathrm{~K}$ and $\mathrm{NO} \nu \mathrm{A}$, which are most efficient in neutrino mode. The $\bar{\nu}$ energies are higher than geoneutrino, reactor neutrino or even spallation neutrino energies, and yet low enough so that atmospheric neutrino backgrounds are small. Unlike beam experiments the $\mu \mathrm{DAR} \bar{\nu}$ energy is also below the muon mass, and so there is no background from charged current $\nu_{\mu}$ or $\bar{\nu}_{\mu}$ interactions. The spectrum is broad enough that such experiments can cleanly distinguish $\delta$ from $\pi-\delta[20,21]$. Finally the main background that appears in water Cherenkov $\mu$ DAR experiments $[2,21]$, the invisible muons, is not present in a scintillator detector as even low energy muons, via ionization, emit scintillation light.

We have considered a 12 year $\mu \mathrm{DAR}$ run together with 12 years of $\mathrm{NO} \nu \mathrm{A}$ appearance data, half in $\nu$ mode and half in $\bar{\nu}$ mode, defined as in ref. [22]. The main background comes from atmospheric $\nu_{e}$ and $\bar{\nu}_{e}$ interacting via IBD and also charged current quasielastic interactions. We adapt the shape of the spectrum of this background from ref. [21]. In that paper we used the unoscillated atmospheric neutrino fluxes at Kamioka in Japan as given in ref. [23] at energies above $100 \mathrm{MeV}$ and at lower energies we used those available at [24]. We expect that at energies below about $200 \mathrm{MeV}$ these fluxes will be about $10 \%$ lower in Guangdong due to the stronger horizontal geomagnetic field, which deflects the low energy cosmic rays responsible for these neutrinos. For each angular bin we then computed the distance that the neutrinos travel and then we folded the neutrino spectra with the corresponding oscillation probabilities. Finally we used GENIE [25] simulations to calculate the inverse $\beta$ decay rate for $\bar{\nu}_{e}$ with protons in the detector and also the CCQE interactions of $\nu_{e}$ and $\bar{\nu}_{e}$ with oxygen in the detector during a 6 year run. We found 26 events between 30 and $55 \mathrm{MeV}$, which is quite consistent with measurements of this background by Super-Kamiokande [26].

Although in the present proposal our target volume is largely carbon, instead of oxygen, we have made the crude estimate that the cross sections per mass are identical and so have simply rescaled the number of events to 50 in a 12 year run while preserving the shape of the spectrum. This is a somewhat pessimistic estimate as our target volume is $10 \%$ smaller. Note that in other locations where similar experiments have been proposed, such as the South Dakota or northern Europe, the background rate will be higher, as the weaker horizontal geomagnetic field leads to nearly a doubling in the low energy neutrino flux. We summarize the expected signal and backgrounds in figure 3 .

Neutral current (NC) atmospheric neutrino events also contribute to the background. Many of these events create neutrons [27] via interactions of atmospheric neutrinos with 


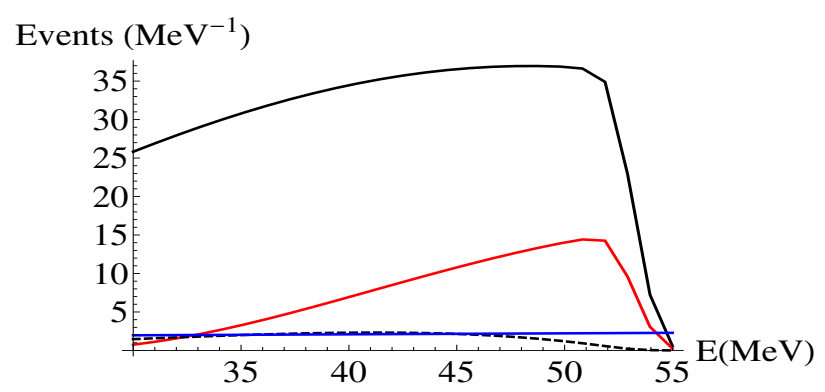

Figure 3. The expected 12 year $\mu \mathrm{DAR}$ IBD event spectrum in a 20 kton liquid scintillator detector at $5 \mathrm{~km}$ (black) and $25 \mathrm{~km}$ (red) assuming $\delta=0^{\circ}$. The blue curve is the atmospheric $\nu_{e}$ background while the black dashed curve is the $\mu^{-}$DAR beam-on background expected at $5 \mathrm{~km}$. The $\mu^{-}$DAR background at $25 \mathrm{~km}$ is considered in our simulations but its effect on the sensitivity is negligible.

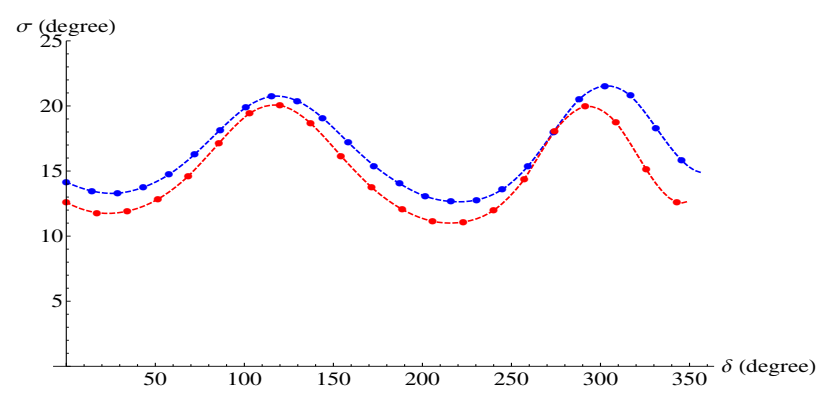

Figure 4. The $1 \sigma$ precision with which $\delta$ can be measured, for each true value of $\delta$, by a 12 year $\mu \mathrm{DAR}$ run with Daya Bay near detectors and 20 kton liquid scintillator detectors at $5 \mathrm{~km}$ and 25 $\mathrm{km}$. 12 years of appearance data at $\mathrm{NO} \nu \mathrm{A}$, as defined in ref. [22], is also used. The red curve does not include the atmospheric neutrino background while the blue curve does. The dots are our numerical results, whereas the curve is an interpolation.

${ }^{12} \mathrm{C}$ in the scintillator. About $40 \%$ create ${ }^{11} \mathrm{C}$ whose eventual decay can be used to veto this background [28], but the remaining background may be comparable to or somewhat larger than our signal in the 30 to $55 \mathrm{MeV}$ regime of interest. It can be measured during beam-off periods, but nonetheless the statistical fluctuations in this background rate would provide a significant source of error if not vetoed.

This background has been evaluated for massive liquid scintillator detectors in ref. [28]. The authors conclude that a pulse shape analysis can allow an $80 \%$ reduction in the NC background at the price of only $10 \%$ of the IBD signal. In the present study this background has not been included, but its rejection is now under investigation.

With two 20 kton detectors, one at $5 \mathrm{~km}$ and another at $25 \mathrm{~km}$, the precision with which $\delta$ can be measured is given in figure 4 . The precision, about $15^{\circ}$, is competitive with other future proposals such as DUNE [29] and Hyper-Kamiokande [30]. Even with a single detector, as can be seen in figure 5 , a precision of about $25^{\circ}$ can be expected.

\section{Conclusions}

The reactor neutrinos of tomorrow will be $30-50 \mathrm{MeV} \bar{\nu}_{\mu}$ with a Michel spectrum, supplied for free by accerator driven subcritical reactors via $\mu \mathrm{DAR}$. Unlike the $\bar{\nu}_{e}$ from conventional 


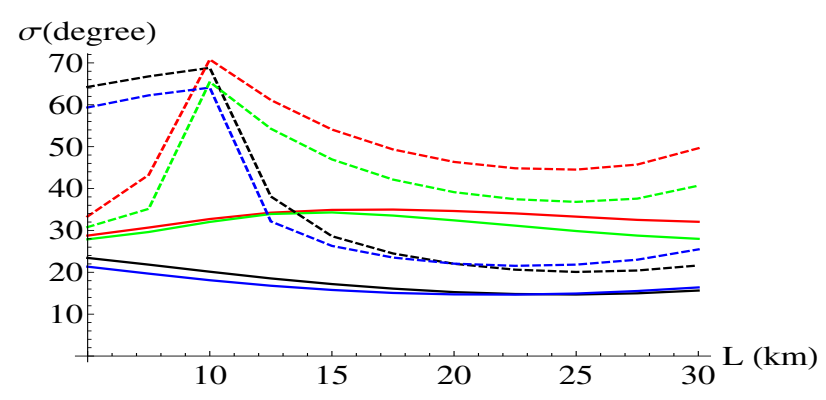

Figure 5. The $\delta$-averaged $1 \sigma$ precision with which $\delta$ can be measured by $\mu \mathrm{DAR}$ with a single 20 kton detector as a function of baseline. The true values of $\delta$ are $0^{\circ}$ (black), $90^{\circ}$ (red), $180^{\circ}$ (blue) or $270^{\circ}$ (green). Solid curves include $\mathrm{NO} \nu \mathrm{A}$.

reactors, they can be used for neutrino experiments in the appearance channel. Such experiments are sensitive to the CP-violating phase $\delta$ and also can directly test the LSND anomaly. On the way to constructing the powerful accelerators needed for these reactors, less powerful but in some cases equally intense accelerators will be built. Some of these will already supply $\bar{\nu}_{\mu}$. At lower energies, a target station can be constructed in which the beam creates unstable isotopes whose decays produce $\bar{\nu}_{e}$, which in turn can be used for disappearance experiments such as sterile neutrino searches.

In particular, with a $10 \mathrm{~mA}, 200 \mathrm{MeV}$ deuteron beam one can obtain a $3 \sigma$ upper bound on the disappearance caused by a $1 \mathrm{eV}$ sterile neutrino of $\sin ^{2}\left(2 \theta_{\mathrm{ee}}\right) \leq 0.02$ in at most 5 years. With a $10 \mathrm{~mA}, 800 \mathrm{MeV}$ proton beam in 5 years one can obtain a $3 \sigma$ upper bound on the appearance caused by such a neutrino of $\sin ^{2}\left(2 \theta_{\mathrm{ee}}\right) \leq 2 \times 10^{-4}$, easily covering the region favored by LSND. In 12 years, with 1 (2) 20 kton liquid scintillator detector(s) one can measure $\delta$ with a precision of $25^{\circ}\left(15^{\circ}\right)$.

\section{Acknowledgments}

JE and EC are supported by NSFC grant 11375201. EC is also supported by the Chinese Academy of Sciences President's International Fellowship Initiative grant 2015PM063.

Open Access. This article is distributed under the terms of the Creative Commons Attribution License (CC-BY 4.0), which permits any use, distribution and reproduction in any medium, provided the original author(s) and source are credited.

\section{References}

[1] Z. Li et al., Physics design of an accelerator for an accelerator-driven subcritical system, Phys. Rev. ST Accel. Beams 16 (2013) 080101 [InSPIRE].

[2] J. Alonso et al., Expression of Interest for a Novel Search for CP-violation in the Neutrino Sector: DAEdALUS, arXiv:1006.0260 [INSPIRE].

[3] J. Cao et al., Muon-decay medium-baseline neutrino beam facility, Phys. Rev. ST Accel. Beams 17 (2014) 090101 [arXiv: 1401.8125] [INSPIRE]. 
[4] OscSNS collaboration, M. Elnimr et al., The OscSNS White Paper, arXiv:1307.7097 [INSPIRE].

[5] ESSnuSB collaboration, E. Baussan et al., A very intense neutrino super beam experiment for leptonic CP-violation discovery based on the European spallation source linac, Nucl. Phys. B 885 (2014) 127 [arXiv:1309.7022] [InSPIRE].

[6] S.V. Bulanov, T. Esirkepov, P. Migliozzi, F. Pegoraro, T. Tajima and F. Terranova, Neutrino oscillation studies with laser-driven beam dump facilities, Nucl. Instrum. Meth. A 540 (2005) 25 [hep-ph/0404190] [INSPIRE].

[7] Yu.S. Lutostansky and V.I. Lyashuk, Antineutrino spectrum from a powerful reactor and neutrino converter system, Phys. Part. Nucl. Lett. 2 (2005) 226 [Pisma Fiz. Elem. Chast. Atom. Yadra 127N4 (2005) 60] [INSPIRE].

[8] A. Bungau et al., Proposal for an Electron Antineutrino Disappearance Search Using High-Rate ${ }^{8}$ Li Production and Decay, Phys. Rev. Lett. 109 (2012) 141802 [arXiv:1205.4419] [INSPIRE].

[9] F. Zhao, Y. Li, C. Han, Q. Fu and X. Chen, IsoDAR Neutrino Experiment Simulation with Proton and Deuteron Beams, arXiv:1509.03922 [INSPIRE].

[10] R. Alba et al., Measurement of neutron yield by 62 MeV proton beam on a thick Beryllium target, J. Phys. Conf. Ser. 420 (2013) 012162 [arXiv:1208.1713] [InSPIRE].

[11] N. Pauwels et al., Experimental determination of neutron spectra produced by bombarding thick targets: deuterons $(100 \mathrm{MeV} / \mathrm{u})$ on ${ }^{9} \mathrm{Be}$ and ${ }^{238} \mathrm{U}$ and ${ }^{36} \mathrm{Ar}$ on ${ }^{12} \mathrm{C}, \mathrm{Nucl}$. Inst. Meth. B160 (2000) 315.

[12] V.I. Lyashuk and Yu.S. Lutostansky, Intensive neutrino source on the base of lithium converter, arXiv:1503.01280 [INSPIRE].

[13] J. Kopp, P.A.N. Machado, M. Maltoni and T. Schwetz, Sterile Neutrino Oscillations: The Global Picture, JHEP 05 (2013) 050 [arXiv: 1303.3011] [INSPIRE].

[14] P. Vogel, L. Wen and C. Zhang, Neutrino Oscillation Studies with Reactors, Nature Commun. 6 (2015) 6935 [arXiv:1503.01059] [INSPIRE].

[15] LSND collaboration, A. Aguilar-Arevalo et al., Evidence for neutrino oscillations from the observation of anti-neutrino(electron) appearance in a anti-neutrino(muon) beam, Phys. Rev. D 64 (2001) 112007 [hep-ex/0104049] [INSPIRE].

[16] ICARUS collaboration, M. Antonello et al., Search for anomalies in the $\nu_{e}$ appearance from a $\nu_{\mu}$ beam, Eur. Phys. J. C 73 (2013) 2599 [arXiv:1307.4699] [InSPIRE].

[17] LSND collaboration, C. Athanassopoulos et al., Measurements of the reactions ${ }^{12} C\left(\nu_{e}, e^{-}\right){ }^{12} N_{\text {g.s. }}$ and ${ }^{12} C\left(\nu_{e}, e^{-}\right)^{12} N^{*}$, Phys. Rev. C 55 (1997) 2078 [nucl-ex/9705001] [INSPIRE].

[18] LSND collaboration, L.B. Auerbach et al., Measurements of charged current reactions of muon neutrinos on ${ }^{12}$ C, Phys. Rev. C 66 (2002) 015501 [nucl-ex/0203011] [INSPIRE].

[19] Y.-F. Li, J. Cao, Y. Wang and L. Zhan, Unambiguous Determination of the Neutrino Mass Hierarchy Using Reactor Neutrinos, Phys. Rev. D 88 (2013) 013008 [arXiv:1303.6733] [INSPIRE].

[20] E. Ciuffoli, J. Evslin and X. Zhang, The Leptonic CP Phase from Muon Decay at Rest with Two Detectors, JHEP 12 (2014) 051 [arXiv:1401.3977] [INSPIRE]. 
[21] J. Evslin, S.-F. Ge and K. Hagiwara, The Leptonic CP Phase from T2(H)K and $\mu^{+}$Decay at Rest, arXiv: 1506.05023 [INSPIRE].

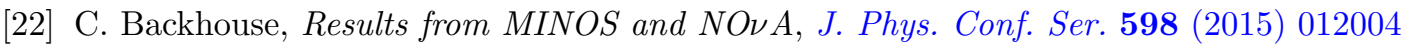
[arXiv: 1501.01016] [INSPIRE].

[23] M. Sajjad Athar, M. Honda, T. Kajita, K. Kasahara and S. Midorikawa, Atmospheric neutrino flux at INO, South Pole and Pyhasalmi, Phys. Lett. B 718 (2013) 1375 [arXiv: 1210.5154] [INSPIRE].

[24] http://www.icrr.u-tokyo.ac.jp/ mhonda/nflx2014/lowe/.

[25] C. Andreopoulos et al., The GENIE Neutrino Monte Carlo Generator, Nucl. Instrum. Meth. A 614 (2010) 87 [arXiv:0905.2517] [INSPIRE].

[26] Super-Kamiokande collaboration, K. Bays et al., Supernova Relic Neutrino Search at Super-Kamiokande, Phys. Rev. D 85 (2012) 052007 [arXiv:1111.5031] [INSPIRE].

[27] KamLAND collaboration, A. Gando et al., A study of extraterrestrial antineutrino sources with the KamLAND detector, Astrophys. J. 745 (2012) 193 [arXiv:1105.3516] [INSPIRE].

[28] R. Möllenberg, F. von Feilitzsch, D. Hellgartner, L. Oberauer, M. Tippmann, V. Zimmer et al., Detecting the Diffuse Supernova Neutrino Background with LENA, Phys. Rev. D 91 (2015) 032005 [arXiv: 1409.2240] [INSPIRE].

[29] M. Goodman, The Deep Underground Neutrino Experiment, Adv. High Energy Phys. 2015 (2015) 256351 [INSPIRE].

[30] Hyper-Kamiokande proto-collaboration, K. Abe et al., Physics potential of a long-baseline neutrino oscillation experiment using a J-PARC neutrino beam and Hyper-Kamiokande, PTEP 2015 (2015) 053C02 [arXiv:1502.05199] [INSPIRE]. 